Geograficando, vol. 14, n. ${ }^{\circ}$, e037, junio 2018. ISSN 2346-898X

Universidad Nacional de La Plata.

Facultad de Humanidades y Ciencias de la Educación.

Departamento de Geografía

\title{
Confort climático del sector norte y sur de la ciudad de Bahía Blanca
}

\section{María Alejandra Ramborger}

Universidad Nacional del Sur, Argentina

alejandra.ramborger@uns.edu.ar

\author{
Alicia María Campo \\ Universidad Nacional del Sur - CONICET, Argentina \\ amcampo@uns.edu.ar
}

\section{María Amalia Lorda}

Universidad Nacional del Sur, Argentina

malorda@criba.edu.ar

Cita sugerida: Ramborger, M. A., Campo, A. y Lorda, M. A. (2018). Confort climático del sector norte y sur de la ciudad de Bahía Blanca. Geograficando 14 (1), e037. https://doi.org/10.24215/2346898Xe037

Recidido: 9 de noviembre de 2017 - Aceptado: 24 de abril de 2018 - Publicado: 31 de julio de 2018 


\title{
Confort climático del sector norte y sur de la ciudad de Bahía Blanca.
}

Climate confort of the northern and southern sector of the city of Bahia Blanca

\author{
Maria Alejandra Ramborger \\ Universidad Nacional del Sur, Argentina \\ alejandra.ramborger@uns.edu.ar \\ Alicia María Campo \\ Universidad Nacional del Sur - CONICET, Argentina \\ amcampo@uns.edu.ar \\ Maria Amalia Lorda \\ Universidad Nacional del Sur, Argentina \\ malorda@criba.edu.ar
}

\section{RESUMEN:}

En la ciudad de Bahía Blanca no se realizaron aún estudios comparativos sobre la calidad ambiental de sus distintos sectores. Por ello se planteó la organización de un Índice de Calidad Ambiental Urbana (ICAU), que contemple dimensiones del soporte físiconatural y del hábitat urbano. Entre los indicadores analizados se encuentra el confort climático. Por las características climáticas de la ciudad, en la cual existe una marcada estacionalidad, debió aplicarse un índice para los meses de verano y otro para los de invierno. Con este trabajo se pretende obtener información para el cálculo del ICAU. Si bien quedan muchos indicadores por evaluar, teniendo en cuenta este análisis podría determinarse que la calidad ambiental del sector norte sería superior a la de la zona sur, ya que la diferencia en el índice aplicado para el período invernal fue más destacada.

Palabras Clave: Confort climático; Calidad ambiental urbana; Bahía Blanca.

\section{Abstract:}

In the city of Bahía Blanca, comparative studies on the environmental quality of the different sectors have not been carried out yet. For this reason, it was proposed the organization of an Urban Environmental Quality Index (UEQI), which contemplates dimensions of the physical-natural support and the urban habitat. Among the indicators analyzed we found the climatic comfort. Due to the climatic characteristics of the city, in which there is a marked seasonality, an index should be applied for the summer months and another for the winter ones. This paper aims to obtain information for the calculation of the UEQI. Although many indicators remain to be evaluated, taking into account this analysis, it could be determined that the environmental quality of the northern sector is higher than the southern area, since the difference in the applied index for the winter period was more prominent. Palabras claves: Confort climático. Calidad ambiental urbana. Bahía Blanca.

KEYWORDS: Climatic comfort; Urban environmental quality; Bahía Blanca.

\section{INTRODUCCIÓN}

Las ciudades son las porciones del territorio más transformadas por la sociedad. En ellas, como resultado de deficiencias en las relaciones entre el subsistema social y natural, suelen aparecer diversos problemas socio ambientales. Estos influyen diferencialmente sobre los distintos sectores del ámbito urbano, por lo que consecuentemente se presentan desigualdades en la calidad ambiental de la población urbana y periurbana. Según Pérez Maldonado (1996),

la calidad ambiental urbana conjuga nociones de propósitos de calidad de vida a nivel urbano y condicionamientos del entorno natural para expresar percepciones de armonía entre lo natural y lo construido (citado por Belkis Cartay, 2004, p. 498).

Mientras que para García Alvarado (1997, p. 14) se trata de 
una adecuación de los elementos que contiene el mismo y las funciones que desarrolla, de manera que el rendimiento sea el considerado óptimo con referencia a un conjunto de valores que la sociedad de manera generalizada admita como positivos.

Un poco más abarcativa es la definición del Grupo de Investigación sobre Calidad Ambiental Urbana (GICAU-FAAULA), la cual la caracteriza como

\begin{abstract}
adecuadas condiciones existentes en un espacio físico urbano, resultante de las apreciables características originales de tales espacios y/o de la apropiada intervención humana de los mismos. Ella produce imágenes, funcionalidades y comportamientos acordes con las dinámicas, preferencias e inquietudes de los usuarios y las capacidades de soporte del mencionado espacio. Todo ello propicia sensaciones de agrado y bienestar de los habitantes y visitantes del espacio en cuestión (citado por Belkis Cartay, 2004, p. 499).
\end{abstract}

De las diversas definiciones consultadas se desprende que la calidad ambiental urbana depende de las condiciones de sitio de la ciudad, la forma en que se ha producido la ocupación de ese espacio y los servicios que brinda la misma. También que influye en gran medida la percepción que se tenga de la oferta de bienes e infraestructuras que el asentamiento ofrece.

Este concepto ha sido abordado en numerosos estudios en los últimos años. García Almirall (2000) realizó una evaluación de la calidad ambiental de un sector del Eixample de Barcelona desde una perspectiva económica utilizando el soporte de herramientas SIG. Escobar (2006) también recurrió a dicho instrumento y lo empleó en la construcción de un índice de calidad ambiental para la ciudad de Cali (Colombia). Cabrera Carranza et al. (2002) estudiaron las relaciones entre calidad ambiental y calidad de vida en la ciudad de Lima a través de diversos índices y arribaron a la conclusión de que los factores ambientales influyen determinantemente en la calidad de vida.

En la ciudad de Bahía Blanca recientemente se produjo un cambio en las demandas locacionales para uso residencial. La población perteneciente a los estratos medios y altos buscó asentarse lejos del ruido, del centro y del movimiento, y acercarse a un entorno más natural, con mayor calidad ambiental. Esto generó una valorización de las áreas periurbanas como lugar de asentamiento. En el imaginario de la población de Bahía Blanca se presentan importantes diferencias respecto a la calidad ambiental que revisten el sector periurbano norte y sur de la ciudad. Este supuesto determinó una mayor expansión en el periurbano norte de las residencias de calidad media y alta, mientras que en el sector sur de la ciudad predomina el asentamiento de población de un nivel socioeconómico medio-bajo.

En la ciudad de Bahía Blanca no se realizaron aún estudios comparativos sobre la calidad ambiental de los distintos sectores de la ciudad, aunque hay numerosos trabajos que abordan algunas de las dimensiones que esta comprende. Zinger et al. (1990), a partir del análisis de los elementos que componen el medio físico, determinaron las zonas con mayor y menor aptitud para la urbanización. Prieto $(2007,2008)$, Prieto y Formiga (2009), y Prieto, Formiga y Medus (2011), si bien incorporaron la dimensión ambiental en sus estudios sobre la calidad de vida del aglomerado urbano de Bahía Blanca, solo tomaron como indicadores para esta variable la recolección de residuos domiciliarios y espacios verdes. Gentili et al. (2012) estudiaron la calidad ambiental en un sector específico del periurbano norte denominado "Cueva de los Leones", donde promotores inmobiliarios privados gestionaron la creación de una reserva natural urbana. Campos y Zinger (2003) realizaron un estudio comparativo, pero, en este caso, de la calidad paisajística de distintos sectores del periurbano norte de la ciudad.

Se plantean como objetivos del presente trabajo la presentación de un Índice de Calidad Ambiental Urbana (ICAU) para la ciudad de Bahía Blanca, que contemple tanto dimensiones propias del soporte físiconatural, como también del hábitat urbano netamente, a fin de determinar las desigualdades existentes en la calidad ambiental urbana de los sectores norte y sur de la ciudad de Bahía Blanca. También se compararán los índices de confort para invierno y verano de los sectores norte y sur de la mencionada localidad. Se pretende de esta manera obtener información sintetizada y elaborada para el cálculo del ICAU, cuya finalidad es facilitar la toma de decisiones y contribuir a la construcción de una estructura espacial que permita un desarrollo eficaz y equitativo de toda la población bahiense. 


\section{Área de ESTUdio: ciUdad de BaHÍa Blanca}

La ciudad de Bahía Blanca, cabecera del partido bonaerense del mismo nombre, se localiza en el suroeste de la provincia de Buenos Aires sobre la costa del océano Atlántico, a los $38^{\circ} 43^{\prime}$ sur y $62^{\circ} 16^{\prime}$ oeste (Figura 1 ). El clima de esta ciudad presenta veranos lluviosos y con altas temperaturas e inviernos secos con bajos registros térmicos. Sin embargo, es necesario tener presente que las condiciones climáticas varían según el sector de la ciudad.

En la zona de Ingeniero White la temperatura desciende unos grados debido a la influencia del mar. El efecto moderador del mar se evidencia además en los valores que presenta la amplitud térmica anual (Capelli de Steffens y Campo de Ferreras, 2004). Las precipitaciones también varían de acuerdo con el sector de la ciudad. Los máximos se producen en el área sur debido a su mayor exposición a los sistemas frontales que ingresan por el cuadrante suroeste, así como en el sector norte, producto de su mayor altitud, mientras que los valores más bajos se localizan en el centro de la ciudad, donde se concentra la edificación en altura (Zapperi, 2012).

FIGURA 1

Localización del partido de Bahía Blanca.

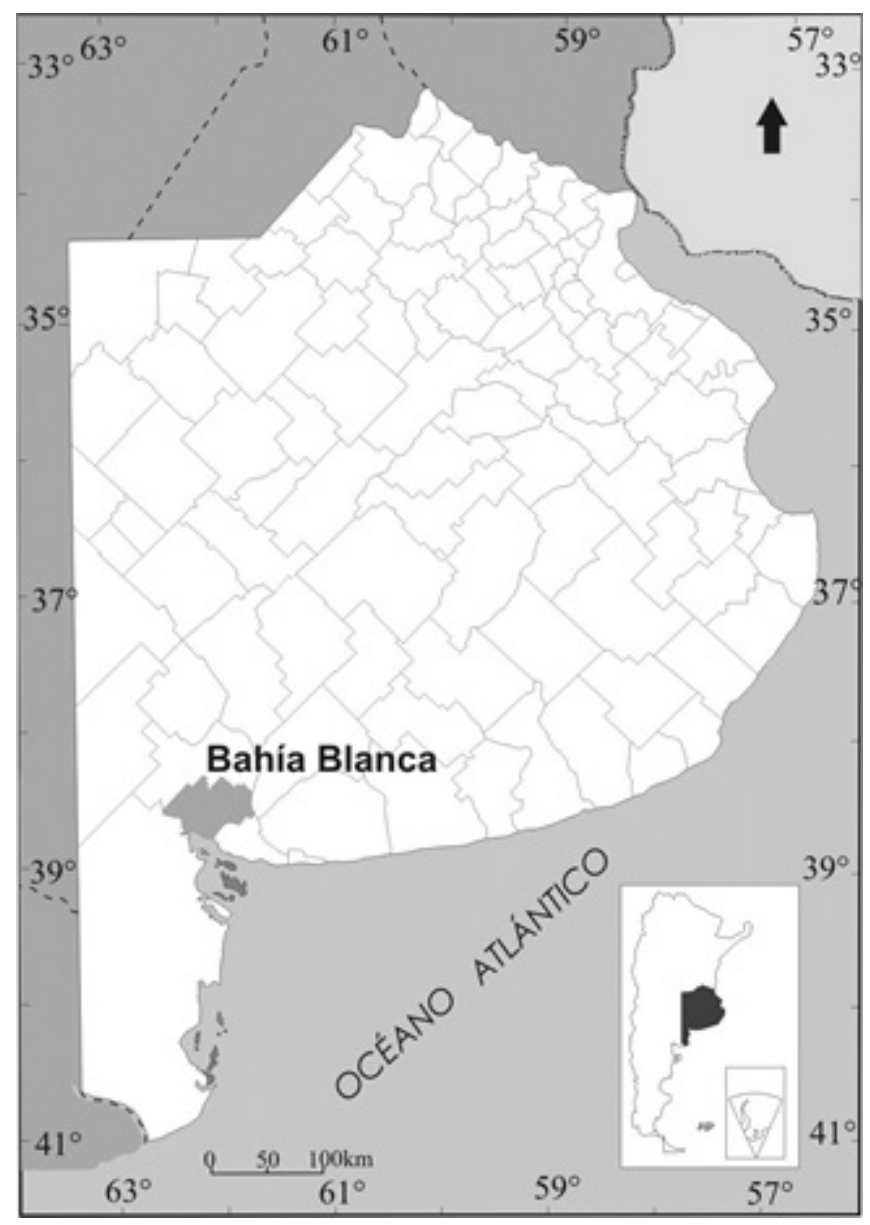

Fuente: Ramborger, 2017.

Dentro del emplazamiento del área urbana es posible distinguir tres unidades geomorfológicas. Al noreste, la llanura meridional de Ventania, que se presenta como una amplia peniplanicie pedemontana de relativa homogeneidad con algunas geoformas menores positivas que adquieren el aspecto de lomas con gradientes suaves ( $1 \%$ a $3 \%)$. La escarpa frontal, que separa esta última unidad de la llanura litoral, se encuentra constituida por un complejo coluvio-aluvio y conos aluviales coalescentes. Sobre el oeste de la misma se 
destacan los conos aluviales de origen fluvial, cuya "coalescencia hace que topográficamente se presenten como llanuras aluviales que morfológicamente funcionan como una bajada” (Zapperi, 2012, p. 33) mientras los de la zona este son producto del movimiento en masa de materiales sobre pendientes. Una vez finalizada esta zona de faldeo se presenta la llanura aluvial del arroyo Napostá, que se extiende hacia el sur y suroeste, y que continúa hacia el sureste uniéndose con la llanura litoral.

En la planicie costera los terrenos presentan escasa altura y, como consecuencia de ello, algunas partes suelen inundarse durante las pleamares excepcionales. Esto genera variaciones en el nivel de la napa freática, lo que se agudiza con la presencia de los vientos del sur.

En el presente estudio se compararán dos sectores de la ciudad: el barrio Parque Norte, ubicado en el sector periurbano norte, y el barrio 26 de Septiembre de la localidad de Ingeniero White, emplazado sobre el sector sur del periurbano de la ciudad sobre la costa del estuario de la bahía Blanca (Figura 2).

\section{MÉtodo de trabajo}

El Índice de Calidad Ambiental Urbana (ICAU) comprende las variables o atributos más representativos de los factores sociales y ambientales del universo bajo análisis los cuales, al integrarse, definen indicadores. Un indicador ambiental es una "variable operacional que señala o describe aspectos o la condición de un fenómeno ambiental que no se puede observar o medir directamente" (Ferraro, 2009, p. 8). A partir de ellos se obtiene el ICAU, el cual resume en una única expresión numérica la información contenida en los múltiples datos recogidos.

El primer paso consistió en identificar las dimensiones que se consideraban de mayor importancia. Luego se seleccionaron indicadores para cada una de ellas. Debido a que la realidad de la ciudad de Bahía Blanca muestra que son pocas las variables disponibles con datos actualizados, y que muchas veces no existe siquiera el registro del indicador, se debió aplicar un método que combina las formas deductiva e inductiva. La primera tiene en cuenta el marco teórico, mientras que la segunda se hace de acuerdo con la disponibilidad de datos. 
FIGURA 2

Ubicación de los sectores bajo análisis

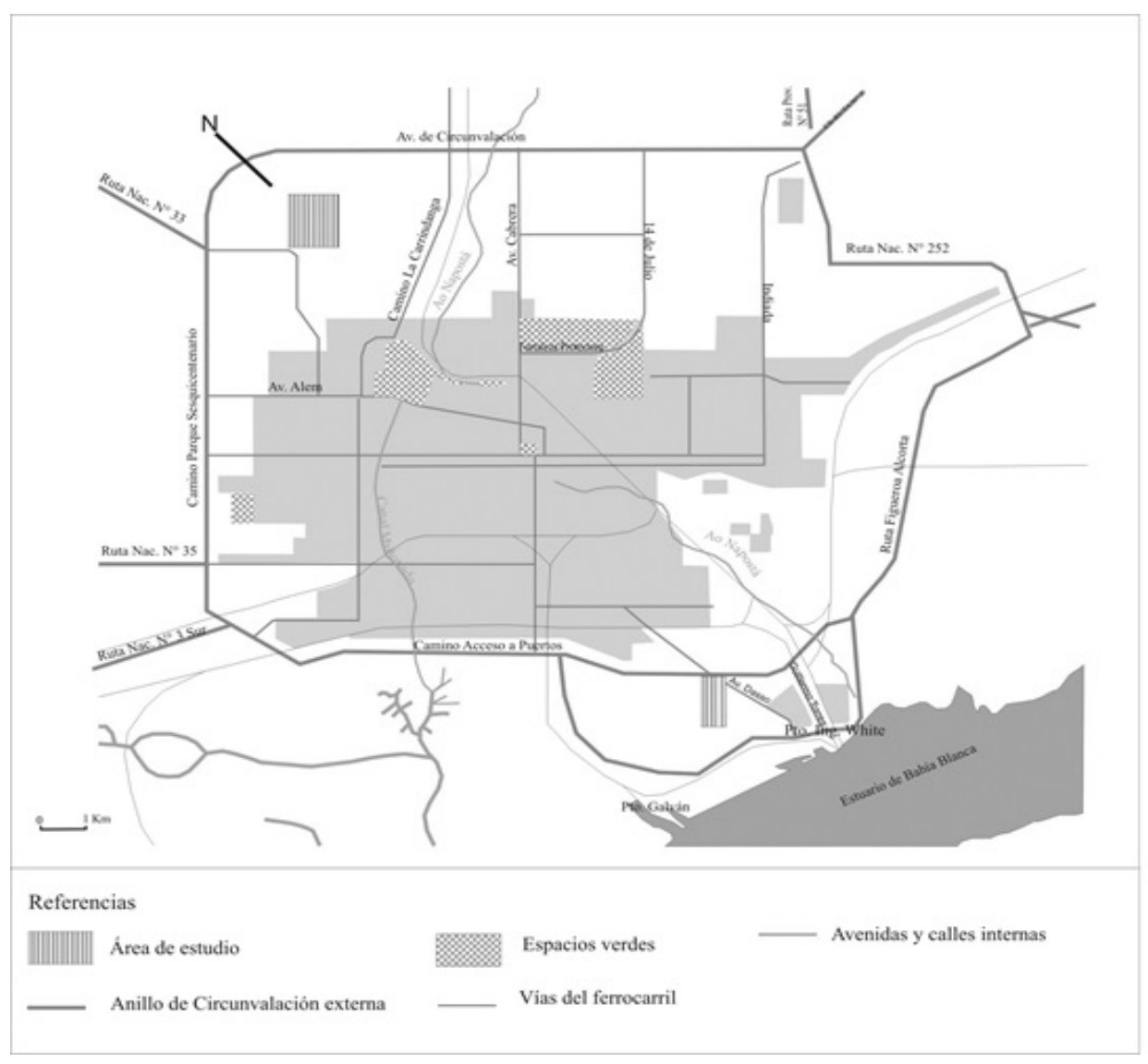

Fuente: Ramborger, 2017 sobre la base de Municipalidad de Bahía Blanca (2000).

El método seleccionado para la construcción del índice es el de ponderación simple, en el que se asume que todos los indicadores de cada dimensión tienen igual importancia, por lo que la ponderación es la misma para cada uno de ellos en función de la cantidad de indicadores que compongan la dimensión. Debido a que los diversos indicadores se encuentran expresados en unidades heterogéneas resultó preciso efectuar su transformación a fin de homogeneizarlas. Para ello se empleó el método estándar de los "puntos de correspondencia" desarrollado en la década de 1970 por el UNRISD (United Nations Research Institute for Social Development).

Los indicadores seleccionados para sintetizar la calidad ambiental urbana de los sectores periurbanos norte y sur de la ciudad de Bahía Blanca se encuentran detallados en la Tabla 1. Los mismos se agruparon en distintas dimensiones e incluyen las variables con máxima relación entre ellas. 
TABLA 1

Índice de Calidad Ambiental Urbana. Dimensiones, indicadores y variables

\begin{tabular}{|c|c|c|}
\hline Dimensión & Indicadores & Variables \\
\hline \multirow{5}{*}{$\begin{array}{l}\text { Soporte } \\
\text { físico- } \\
\text { natural }\end{array}$} & \multirow[b]{2}{*}{ Confort climático } & Enfriamiento \\
\hline & & İndice Termo-higrométrico \\
\hline & \multirow[t]{2}{*}{ Biodiversidad } & $\begin{array}{l}\text { Densidad arbolado urbano (cantidad de } \\
\text { ejemplares por manzana) }\end{array}$ \\
\hline & & Densidad áreas verdes $\left(\mathrm{m}^{2} / \mathrm{hab}\right)$ \\
\hline & Calidad del aire & Valor del pH en el agua de lluvia \\
\hline \multirow{13}{*}{$\begin{array}{l}\text { Hábitat } \\
\text { urbano }\end{array}$} & \multirow{6}{*}{$\begin{array}{l}\text { Consolidación } \\
\text { urbana }\end{array}$} & $\begin{array}{l}\text { Porcentaje de hogares con existencia de energía } \\
\text { eléctrica domiciliaria }\end{array}$ \\
\hline & & $\begin{array}{l}\text { Porcentaje de hogares con existencia de gas de } \\
\text { red }\end{array}$ \\
\hline & & $\begin{array}{l}\text { Porcentaje de hogares con existencia de } \\
\text { alumbrado público }\end{array}$ \\
\hline & & $\begin{array}{l}\text { Porcentaje de hogares con al menos una cuadra } \\
\text { pavimentada }\end{array}$ \\
\hline & & $\begin{array}{l}\text { Porcentaje de hogares que reside en viviendas } \\
\text { œn agua de red }\end{array}$ \\
\hline & & $\begin{array}{l}\text { Porcentaje de hogares que reside en hogares con } \\
\text { inodoro con descarga de agua y desagüe a red }\end{array}$ \\
\hline & Tráfico urbano & Flujo vehicular por minuto \\
\hline & \multirow[t]{2}{*}{ Higiene urbana } & $\begin{array}{l}\text { Porcentaje de población que reside en viviendas } \\
\text { œn servicio regular de recolección de residuos }\end{array}$ \\
\hline & & $\begin{array}{l}\text { Porcentaje de población residente a menos de } \\
300 \text { metros de un basural a cielo abierto }\end{array}$ \\
\hline & Salud & Distancia a los centros de salud más cercanos \\
\hline & \multirow{3}{*}{$\begin{array}{l}\text { Calidad } \\
\text { paisajística }\end{array}$} & Calidad visual intrínseca \\
\hline & & Calidad visual del entorno inmediato \\
\hline & & Calidad del fondo escénico \\
\hline \multirow{4}{*}{ Ambiental } & $\begin{array}{l}\text { Riesgos } \\
\text { naturales }\end{array}$ & $\begin{array}{l}\text { Superficie del territorio en condiciones de riesgo } \\
\text { de inundabilidad por habitante (en hectáreas) }\end{array}$ \\
\hline & \multirow{3}{*}{$\begin{array}{l}\text { Afectación del } \\
\text { medio como } \\
\text { consecuencia } \\
\text { del desarrollo de } \\
\text { actividades } \\
\text { impactantes }\end{array}$} & Importancia del impacto de la actividad ladrillera \\
\hline & & Importancia del impacto del acopio de cereales \\
\hline & & Exposición a contaminación por industrias \\
\hline \multirow{4}{*}{$\begin{array}{l}\text { Vida } \\
\text { comunitaria }\end{array}$} & \multirow{2}{*}{$\begin{array}{l}\text { Seguridad } \\
\text { ciudadana }\end{array}$} & Cantidad de delitos anuales contra la propiedad \\
\hline & & Cantidad de delitos anuales contra las personas \\
\hline & \multirow{2}{*}{$\begin{array}{l}\text { Satisfacción con } \\
\text { la comunidad } \\
\text { local }\end{array}$} & Grado de satisfacción con el barrio para vivir \\
\hline & & Grado de satisfacción con la gestión municipal \\
\hline
\end{tabular}

Fuente: Ramborger, elaborado sobre la base de varios autores 
Entre los indicadores analizados para el soporte físico-natural se encuentra el confort climático. Los parámetros meteorológicos afectan el desarrollo humano en términos termo-fisiológicos, por ello se estimó importante tenerlos en cuenta al momento de analizar la calidad ambiental urbana.

Se considera que existe confort climático o confortabilidad cuando las condiciones climáticas permiten al organismo permanecer en estado de reposo, o cuando los mecanismos de autorregulación que se activan son mínimos, gracias a la existencia de cierto equilibrio energético con el medio (Fernández García, 1994). Mientras que se produce disconfort climático |en aquellas situaciones en las que el cuerpo debe realizar un gran esfuerzo para preservar su estabilidad, lo que causa un gran estrés (Besancenot, 1991 en Sastre, 2009).

El concepto de confort admite diferentes acepciones de acuerdo con la perspectiva desde la cual se lo analice. Desde el punto de vista psicológico, se puede definir como la condición mental en la cual existe satisfacción con el ambiente térmico. Esta conceptualización presenta muchas dificultades para llevarla a la práctica debido a su carácter subjetivo. Desde el aspecto fisiológico, se asocia con la mínima proporción de señales nerviosas desde los receptores térmicos en la piel y el hipotálamo. Mientras que si se considera la definición energética, ésta se lograría cuando los flujos de calor desde y hacia el individuo están balanceados (Ruiz y Correa, 2009).

El confort climático comprende tanto la hidrotermia o confort térmico, que equivale al mantenimiento de la temperatura corporal, como también el confort hídrico. Este último se refiere al intercambio de oxígeno y vapor de agua entre el ambiente exterior y la sangre. Este proceso depende de la presión de vapor de agua presente en la atmósfera. Si dicha variable supera los 31,3 hPa, el vapor de agua excedente en el aire tiende a pasar al plasma y diluirlo, mientras que si es inferior a dicho valor se produce evaporación a expensas del plasma, que en consecuencia se deshidrata.

Las principales variables climáticas que influyen sobre el confort son la temperatura, la humedad, el viento y la radiación. La temperatura es la que afecta de manera más directa la sensación de bienestar, por la alta sensibilidad del organismo humano a sus cambios.

Las altas temperaturas provocan vasodilatación con la consiguiente disminución de la presión arterial periférica y sensación de agotamiento y fatiga por falta de irrigación sanguínea a los músculos y otros órganos vitales. Además obligan al cuerpo a exudar grandes cantidades de líquido con el motivo de disminuir la temperatura superficial de la piel. En este proceso de transpiración el cuerpo pierde mucho agua y electrolitos que causan en el peor de los casos, deshidratación. Las temperaturas muy bajas, por el contrario, aumentan la presión arterial provocando problemas circulatorios que desembocan en trastornos cardíacos (Besancenot en Sastre, 2009, p. 16).

La humedad regula el proceso de evaporación, por lo que desempeña un papel importante cuando se presentan altas temperaturas. La relevancia del proceso mencionado reside en que permite eliminar la transpiración de la superficie de la piel, lo que da lugar a su enfriamiento. Un porcentaje bajo de humedad torna mucho más rápido dicho mecanismo, mientras que si se presentan valores altos el mismo se vuelve lento y difícil, lo que genera malestar en los individuos. Cuando las temperaturas son bajas, por el contrario, aumenta la sensación de disconfort, sobre todo en caso de que el aire está muy seco.

El viento puede ser un elemento favorable, ya que aumenta la pérdida de calor de la piel cuando la temperatura de ésta es superior a la del aire. Por el contrario, cuando el aire se encuentra más caliente que la superficie corporal, el viento aumenta la sensación de calor. Además, es importante tener en cuenta la temperatura radiante del entorno, ya que muchos estudios sugieren que es más significativa que la del aire.

Las sensaciones que el hombre experimenta ante situaciones climáticas similares pueden variar por la influencia de factores individuales de carácter psicológico y físico (edad, peso, constitución física, entre otros), y a la capacidad de adaptación y los mecanismos de defensa que los distintos grupos humanos han ido desarrollando. Sin embargo, es posible encontrar cierta universalidad en la temperatura óptima que todo ser humano requiere para lograr una sensación de bienestar climático. De acuerdo con Moreno Oliver (2002), los parámetros meteorológicos deberían encontrarse en los valores que se presentan en la Tabla 2. 
TABLA 2

Parámetros de confort

\begin{tabular}{|c|c|}
\hline $\begin{array}{c}\text { Condición } \\
\text { atmosférica }\end{array}$ & Valores de confort \\
\hline Temperatura & $20^{\circ} \mathrm{C}$ a $25^{\circ} \mathrm{C}$ \\
\hline Humedad & $40 \%$ a $70 \%$ \\
\hline Velocidad del aire & $0,15 \mathrm{~m} / \mathrm{s} \mathrm{a} 0,25 \mathrm{~m} / \mathrm{s}$ \\
\hline Presión & $1013,2 \mathrm{mb}$ \\
\hline Contaminación & Exenta \\
\hline $\begin{array}{c}\text { Ionización } \\
\text { atmosférica }\end{array}$ & Predominancia iones \\
\hline
\end{tabular}

Moreno Oliver, 2002.

La construcción de las ciudades produce alteraciones sobre la superficie terrestre que repercuten en las capas más bajas de la atmósfera. Como resultado se producen cambios en los diferentes elementos climáticos que definen el clima del lugar. En el caso de la temperatura, en las áreas urbanas es función tanto de la propia radiación solar como del intercambio calórico entre la atmósfera y los distintos materiales empleados en los edificios, calles, etc. Éstos, al estar compuestos principalmente por metales, conducen más rápidamente el calor que la tierra húmeda o los espacios verdes, por lo que todo el flujo de calor absorbido y almacenado a lo largo del día es liberado durante las últimas horas de la tarde. A ello debe añadirse el calor generado por las emisiones de humo y gases calientes, producto del desarrollo de las actividades industriales, los sistemas de calefacción y el parque automotor.

Uno de los procesos que se origina como consecuencia de lo mencionado es la aparición de temperaturas más elevadas en la parte central de las ciudades, conocido como isla de calor. Esta aparece generalmente con condiciones de tiempo calmo y claro. Su intensidad depende de la topografía del lugar, la existencia de espacios verdes, el ancho de calles y avenidas que favorecen el libre desplazamiento del aire, la disposición y altura de los edificios, el trazado urbano y la presencia de partículas en la atmósfera. Además, varía entre el día y la noche y entre el verano y el invierno. El mayor almacenamiento de calor en el suelo también tiene como consecuencia una reducción del número de heladas con respecto al entorno rural, lo cual en este caso genera un cambio de índole beneficioso.

Otra diferencia respecto al clima del área extraurbana es la disminución de la evapotranspiración, producto de una menor superficie de masa vegetal, de cuerpos de agua y de suelo desnudo, y de la reducción de la energía solar necesaria para evaporar el rocío en la mañana, dado que la absorben las paredes de los edificios. Aun así, se producen aumentos periódicos de la humedad relativa, sobre todo por las noches en épocas de buen tiempo y en invierno, como consecuencia de la presencia de núcleos higroscópicos generados por la contaminación. Esto deriva en la formación de nieblas en invierno y de nubosidad en verano, lo cual genera una disminución del número de días de sol y un aumento del régimen de lluvias. Otros factores que inducirían un incremento en el monto de las precipitaciones en las ciudades, según Landsberg (1991), son el movimiento vertical de 
las masas de aire por efecto de la isla de calor y el efecto barrera que ejerce la edificación para el paso de los sistemas frontales.

En las áreas urbanas, la velocidad y dirección de los vientos también suele verse alterada a causa de la rugosidad del suelo, la canalización de éstos en las calles (efecto corredor) y por influencia de la isla de calor, que modifica los gradientes térmicos.

Al circular el aire entre los edificios se genera turbulencia y vórtices que perturban el desplazamiento de los habitantes. Dicho flujo se modifica constantemente en función de la forma, altura y disposición de unos con respecto a los otros (Munn, 1968 en Capelli de Steffens et al, 2005, p. 118).

Las modificaciones en los elementos meteorológicos mencionados generan diferencias microclimáticas dentro de una misma ciudad, lo que produce variaciones en los grados de confort climático dentro de dicho ámbito urbano. La isla urbana de calor mantiene los niveles de temperatura altos por mucho más tiempo, lo que causa que durante un plazo mayor se experimente estrés climático y que se reduzcan los períodos de recuperación de los residentes urbanos.

Para la medición del confort por las características climáticas de la ciudad de Bahía Blanca, en la cual existe una marcada estacionalidad, se consideró conveniente la aplicación de un índice para los meses de verano y otro para los de invierno. Para el caso de la estación estival, se seleccionó el índice termo-higrométrico el cual “indica la temperatura que siente el individuo en ${ }^{\circ} \mathrm{C}$, al considerar la temperatura máxima diaria y la humedad relativa del aire" (Huamantinco Cisneros, 2010, p. 206). Su cálculo se realizó mediante la siguiente fórmula:

$\mathrm{THI}=\mathrm{T}-\left[0,55-\left(0,0055^{*} \mathrm{HR}\right)^{*}(\mathrm{~T}-14,5)\right]$

( $T$ es la temperatura real y HR es la humedad relativa).

Para la obtención del índice térmico de frío se combinan las variables temperatura y velocidad del viento. En el caso del presente trabajo se seleccionó el cálculo de la temperatura equivalente (TE), para ello se empleó la ecuación de Quayle y Steadman (1999), donde T es la temperatura real y V es la velocidad del viento:

$\mathrm{T}_{\mathrm{E}}=1,41-1,162 \mathrm{~V}+0,980 \mathrm{~T}+0,0124 \mathrm{~V}^{2}+0,0185 \mathrm{VT}$

A partir del valor obtenido es posible obtener un factor de enfriamiento, aplicando el siguiente cálculo:

Enfriamiento= Temperatura real - Temperatura equivalente

Los datos para el cálculo fueron obtenidos de las estaciones meteorológicas pertenecientes al Departamento de Geografía y Turismo de la Universidad Nacional del Sur, localizadas en el Club Náutico Bahía Blanca y en el Complejo Alem de la sede universitaria, durante el período junio-agosto del año 2015, diciembre 2015, y enero y febrero del año 2016. 


\section{RESULTADOS Y DISCUSIÓN}

El análisis de los datos meteorológicos correspondiente a los meses de junio, julio y agosto del año 2015 da como resultado una temperatura media mensual de $10,9 \mathrm{oC}$ en el caso de Ingeniero White, mientras que en la zona norte de la ciudad de Bahía Blanca fue ligeramente superior con un valor de $11,1^{\circ} \mathrm{C}$. El mes en que se registró la temperatura más baja fue junio en ambos casos. La sensación térmica fue en promedio 7,1 oC menor que la temperatura registrada para el caso del primer sector, en cambio en el otro solo fue inferior en 2,31 oC.

La humedad relativa en el caso de Ingeniero White presentó valores entre 18 y $99 \%$, con un promedio de $62 \%$. El mes con menor humedad relativa fue junio con una media de $60 \%$. En el sector norte se exhibieron valores entre 19 y $88 \%$ con un promedio del $56 \%$. También fue junio el mes con menor humedad relativa (53\%).

En cuanto al viento, en los momentos más fríos en la localidad de Ingeniero White predominó la dirección norte, seguida del nornoroeste y nornoreste, con una velocidad promedio de $15,3 \mathrm{~km} /$ hora. En el sector norte de la ciudad prevalecieron los vientos de los cuadrantes norte y noreste, con una velocidad media de 7,8 km/ hora. Las temperaturas más bajas se presentaron en el caso de Ing. White en horas de la mañana, entre las 5:30 y las 10:00, éstas coinciden en un $63 \%$ con los mayores porcentajes de humedad del día y en un $50 \%$ con las velocidades más altas del viento. En el caso del sector norte los momentos más fríos del día acontecieron a la noche, entre las 23:00 y 23:50 y concordaron en un $50 \%$ con los mayores porcentajes de humedad y las velocidades más altas del viento.

El período estival se caracterizó en la localidad de Ingeniero White por temperaturas máximas entre 38,8 ${ }^{\circ} \mathrm{C}$ y $18,8{ }^{\circ} \mathrm{C}$, con una media mensual de $23,4^{\circ} \mathrm{C}$, mientras que en el sector norte la temperatura promedio fue levemente superior, con un valor de $23,6^{\circ} \mathrm{C}$. El mes en que se presentó la temperatura máxima fue enero en ambos casos. Los horarios en los cuales se exhibieron las máximas temperaturas se encuentran entre las 15:30 y las 18:30 para los dos sectores. Los vientos predominaron del cuadrante norte, con una velocidad media de $12,9 \mathrm{~km} /$ hora para el caso de Ingeniero White y de $6,3 \mathrm{~km} /$ hora para el sector norte. La humedad relativa media fue $65 \%$ en la localidad de Ing. White, y fue similar durante todos los meses de la estación. En el caso del sector norte, este parámetro presentó un promedio de $52,7 \%$ con poca variabilidad durante la estación en estudio.

El estudio de los índices de confort arrojó como resultado para la estación invernal un factor de enfriamiento superior en el caso de Ingeniero White, de 7,3, producto de valores más elevados de humedad y de velocidad del viento provocados por la influencia del mar adyacente. Mientras que en el caso de la estación estival, resulta más desfavorable el sector norte con un porcentaje más importante de días con disconfort, $81,8 \%$, contra 76,4\% en Ing. White, en donde la acción moderadora del agua atempera la sensación de calor.

\section{Conclusiones}

Se ha presentado la estructura de la información que permitirá obtener el Índice de Calidad Ambiental Urbana, cuyo objetivo es determinar las desigualdades existentes en la calidad ambiental urbana de los sectores norte y sur de la ciudad de Bahía Blanca. Se advirtió que se trata de un proceso complejo pues se requieren datos provenientes de distintos tipos de fuente, lo que trae como dificultad que las metodologías de medición y análisis no son las mismas. A ello se añade el inconveniente de que la información se presenta disgregada de acuerdo con las unidades administrativas que no coinciden con el área bajo análisis. Otro problema es que los datos para las variables ambientales suelen tener fuentes de información dispersas, o en algunos casos inexistentes, a lo que se suma que en muchas ocasiones no son comparables y sus escalas de análisis y características difieren totalmente de las sociales. 
TABLA 3

Datos meteorológicos invierno y verano Ing. White y zona Norte

\begin{tabular}{|c|c|c|}
\hline & Ingeniero White & Zona Norte \\
\hline \multicolumn{3}{|l|}{ Invierno } \\
\hline Enfriamiento & 3,8 & 8,8 \\
\hline Temperatura media $\left({ }^{\circ} \mathrm{C}\right)$ & $10,9^{\circ} \mathrm{C}$ & $11,1^{\circ} \mathrm{C}$ \\
\hline Factor Variación Medio & $-7,1$ & $-2,3$ \\
\hline Temperatura Mínima & $-2,2^{\circ} \mathrm{C}(23 / 06 / 2015)$ & $-4,1^{\circ} \mathrm{C}(23 / 06 / 2015)$ \\
\hline \multicolumn{3}{|l|}{ Verano } \\
\hline $\begin{array}{l}\text { Indice termo- } \\
\text { higrométrico medio }\end{array}$ & $32,4^{\circ} \mathrm{C}$ & $32,3^{\circ} \mathrm{C}$ \\
\hline Temperatura media & $23,4^{\circ} \mathrm{C}$ & $23,6^{\circ} \mathrm{C}$ \\
\hline $\begin{array}{l}\text { Factor de variación } \\
\text { medio }\end{array}$ & $2,3^{\circ} \mathrm{C}$ & $1,8^{\circ} \mathrm{C}$ \\
\hline Temperatura máxima & $38,8^{\circ} \mathrm{C}(21 / 01 / 2016)$ & $38,6^{\circ} \mathrm{C}(27 / 12 / 2015)$ \\
\hline
\end{tabular}

Elaborado por Ramborger sobre la base de los datos obtenidos en las estaciones meteorológicas pertenecientes al Departamento de Geografía y Turismo de la Universidad Nacional del Sur

Se estimó importante iniciar la construcción del ICAU con el análisis del confort climático, ya que los parámetros meteorológicos afectan el desarrollo humano en términos termofisiológicos. Del análisis de este parámetro se pudo detectar que, durante la estación invernal se presenta más confortable la zona norte de la ciudad, en virtud de que el factor de enfriamiento es superior en la zona de Ingeniero White. Esto obedece a que los valores de humedad y de velocidad del viento son superiores en el sector sur como consecuencia de la influencia del mar adyacente. Mientras que en el período estival resulta a la inversa, la zona sur resulta más confortable dado que la acción moderadora del agua atempera la sensación de calor, pero con muy poco margen de diferencia.

Por último, si bien quedan muchos indicadores por analizar, si tenemos en cuenta este primer análisis podría determinarse que la calidad ambiental del sector norte sería superior a la del sector de Ing. White, ya que la diferencia en el índice aplicado para el período invernal fue más destacada.

\section{BiBLiografía}

Belkis Cartay, A. (2004). Consideraciones en torno a los conceptos de calidad de vida y calidad ambiental. Fermentum, Año 14 (41), 491-502. Recuperado de: http://www.saber.ula.ve/bitstream/123456789/20587/2/articulo3.pdf.

Cabrera Carranza, C., Maldonado D., M., Arévalo G., W., Pacheco A., R., Giraldo V., A. y Loayza, S. (2002). Relaciones entre calidad ambiental y calidad de vida en Lima metropolitana. Revista del Instituto de Investigaciones de la Facultad de Geologia, Minas, Metalurgia y Ciencias Geográfica, 5 (9), 47-52. Recuperado de:http://revistasinve stigacion.unmsm.edu.pe/index.php/iigeo/article/view/664/518 
Campos, M. y Zinger, S. (2003). Calidad paisajística y recreación. El periurbano norte de Bahía Blanca. Condet, Año II, Vol. III, 21-34. Recuperado de: http://www.condet.edu.ar/cndt/images/articulos/Articulo\%202\%20Camp os\%20Zinger\%20Condet\%203.pdf

Capelli de Steffens, A. y Campo de Ferreras, A. (2004). Climatología. En M. C. Piccolo y M. S. Hoffmeyer (Eds.), Ecosistema del Estuario de Bahía Blanca (pp. 79-90). Bahía Blanca: Instituto Argentino de Oceanografía,

Capelli de Stefens, A., Piccolo, C., y Campo de Ferreras, A. (2005). Clima urbano de Babía Blanca. Bahía Blanca: Universidad Nacional del Sur, Departamento de Geografía y Turismo.

Escobar, L. (2006). Indicadores sintéticos de calidad ambiental. Un modelo general para grandes zonas urbanas. Eure, 32 (96), 73-98. Recuperado de: www.scielo.cl/scielo.php? pid $=$ S0250-71612006000200005\&script $=$ sci_arttext\&tlng=en

Fernández García, F. (1994). Clima y confortabilidad humana. Aspectos metodológicos. Serie Geográfica, 4,109-125. Recuperado de: http://dspace.uah.es/dspace/bitstream/handle/10017/1030/Clima\%20y\%20Confortabilidad \%20Humana.\%20Aspectos\%20Metodol\%C3\%B3gicos.pdf?sequence $=1$

Ferraro, R. (2009). Material Cátedra Ecología Urbana y periurbana, Maestría en Gestión Ambiental del Desarrollo Urbano, Facultad de Arquitectura, Diseño y Urbanismo, Universidad Nacional de Mar del Plata, Mar del Plata, Argentina.

García Almirall, M. P. (2000). Un SIG para la evaluación ambiental de la ciudad. Sitnat, Portal de Conocimiento y Participación. Recuperado de: ww2.pcypsitna.navarra.es/C4/Territorial\%202000/Document\%20Library/ COMUNICA/EVALUACION\%20AMBIENTAL.pdf

García Alvarado, J. M. (1997). Propuesta teórica-metodológica para la valoración de la calidad urbano ambiental (QT).Anales de geografía de la Universidad Complutense, 17. Recuperado de:revistas.ucm.es/index.php/AGUC/ article/download/AGUC9797110011A/31431

Gentili, J., Gil, V., Campo, A. M., y Rosell, P. (2012). Calidad ambiental en "Cueva de los leones", Periurbano de Bahía Blanca, Argentina. Geografia y Sistemas de Información Geográfica (GEOSIG), 4 (4) Sección I, 265-278. Recuperado de: http://www.gesig-proeg.com.ar/documentos/revista-geosig/2012/Investigacion/12-GENTIL I-ETAL-GEOSIG4-2012B.pdf

Huamantinco Cisneros, M. A., y Piccolo, M. C. (2010). Índices de confort aplicados al balneario Monte Hermoso, Argentina. Investigaciones geográficas, 52, 201-214.

Landsberg, H. E. (1981). The Urban Climate. New York: Academic Press.

Moreno Oliver, F. X. (2002). Meteoropedagogía. Revista de ciéncies de l'educació. Universitas Tarraconensis, nº 1, pág. 93-102.

Municipalidad de Bahía Blanca (2000). Plan Estratégico Bahía Blanca. Etapa de formulación Diciembre 1997diciembre 1999. Documento Final. Bahía Blanca, Argentina: Municipalidad de Bahía Blanca, Dirección de Planificación Estratégica.

Prieto, M. B. (2007). Condiciones habitacionales y calidad de vida. El caso de Bahía Blanca. X Jornadas Argentinas de Estudios de Población 2009. Recuperado de: http://www.produccion.fsoc.uba.ar/aepa/ixjornadas/pdf/69.pdf

Prieto, M. B. (2008). Fragmentación socio-territorial y la calidad de vida en Bahía Blanca. Geograficando, 4 (4), 193-214. Recuperado de: http://www.memoria.fahce.unlp.edu.ar/art_revistas/pr.3746/pr.3746.pdf

Prieto, M. B. y Formiga, N. (2009). Localización y accesibilidad: incidencia en la calidad de vida de los adultos mayores en la ciudad de Bahía Blanca. X Jornadas Argentinas de Estudios de Población 2009. Recuperado de: http://w ww.produccion.fsoc.uba.ar/aepa/xjornadas/pdf/69.pdf

Prieto, M. B., Formiga, N., y Medus, S. (2011). La segregación socio-residencial en ciudades intermedias. El caso de Bahía Blanca - Argentina. XIII Conferencia Iberoamericana de Sistemas de Información Geográfica. Recuperado de: http://www.inegi.org.mx/eventos/2011/conf_ibero/doc/ET4_19_PRIETO.pdf

Ruiz, M. A., y Correa, E. N. (2009). Confort térmico en espacios abiertos. Comparación de modelos y su aplicabilidad en ciudades de zonas áridas. Avances en Energías Renovables y Medio Ambiente, 13, pag. 71-78. Recuperado de: http://www.asades.org.ar/modulos/averma/trabajos/2009/2009-t001-a010.pdf 
Sastre, B. (2009). Aptitudes climatoterapéuticas de Villa Ventana, partido de Tornquist, provincia de Buenos Aires (Tesis de licenciatura). Departamento de Geografía y Turismo, Universidad Nacional del Sur, Bahía Blanca, Argentina.

Zapperi, P. (2012). Hidrografía urbana de Babia Blanca (Tesis doctoral). Universidad Nacional del Sur, Bahía Blanca, Argentina.

Zinger, S., Del Pozo, O., y De Gaettano, R. (1990). Bahía Blanca: análisis de la aptitud del medio natural para la expansión urbana. Revista Universitaria de Geografía, 4 (1), 79-97. 